OPEN ACCESS

Edited by:

Laurent M. Sachs,

Muséum National d'Histoire Naturelle,

France

Reviewed by:

Veerle M. Darras,

KU Leuven, Belgium

Paul Webb,

California Institute for Regenerative

Medicine, United States

*Correspondence:

Aurea Orozco

aureao@unam.mx

†These authors have contributed equally to this work

Specialty section: This article was submitted to Thyroid Endocrinology, a section of the journa

Frontiers in Endocrinology

Received: 08 December 2018 Accepted: 21 February 2019

Published: 14 March 2019

Citation:

Lazcano I, Rodríguez-Ortiz $R$ Villalobos $P$, Martínez-Torres A, Solis-Saínz JC and Orozco A (2019)

Knock-Down of Specific Thyroid Hormone Receptor Isoforms Impairs Body Plan Development in Zebrafish.

Front. Endocrinol. 10:156

doi: 10.3389/fendo.2019.00156

\section{Knock-Down of Specific Thyroid Hormone Receptor Isoforms Impairs Body Plan Development in Zebrafish}

\author{
Iván Lazcano ${ }^{1,2 \dagger}$, Roberto Rodríguez-Ortiz ${ }^{1,3 \dagger}$, Patricia Villalobos ${ }^{1}$, \\ Ataúlfo Martínez-Torres ${ }^{1}$, Juan Carlos Solís-Saínz ${ }^{2}$ and Aurea Orozco ${ }^{1 *}$ \\ ${ }^{1}$ Departamento de Neurobiología Celular y Molecular, Instituto de Neurobiología, Universidad Nacional Autónoma de México \\ (UNAM), Querétaro, Mexico, ${ }^{2}$ Departmento de Investigación Biomédica, Facultad de Medicina, Universidad Autónoma de \\ Querétaro, Querétaro, Mexico, ${ }^{3}$ CONACYT - Instituto de Neurobiología, Universidad Nacional Autónoma de México, \\ Querétaro, Mexico
}

The role of thyroid hormones (THs) in development has been extensively studied, however, the specific molecular mechanisms involved are far from being clear. THs act by binding to TH nuclear receptors (TR) that act as ligand-dependent transcription factors to regulate $\mathrm{TH}$-dependent gene expression. Like vertebrates, zebrafish express different isoforms of functional $\mathrm{Tr}$ alpha and beta, some of which can bind alternative ligands like 3,5-T2. In this study, we first analyzed the effects of exogenous T3 and 3,5-T2 exposure during embryogenesis. The percentage of affected embryos was similar to those vehicle-injected, suggesting that the early exposure to low TH levels is not sufficient to elicit effects upon the phenotype of the embryo. We then generated crispants for four isoforms of thr to learn more about the role of these receptors in early development. We found that crispant larvae from thraa and a newly identified l-thrb+, but not thrab and canonical thrb1 showed profound deleterious effects upon symmetry and laterality, suggesting early novel roles for these $\mathrm{Tr}$ isoforms in the body plan developmental program. Since critical events that determine cell fate start in the late gastrula, we tested if some genes that are expressed during early developmental stages could indeed be $\mathrm{TH}$ targets. We identify early development genes, like sox10 and eve, that were specifically over-expressed in thraa and I-thrb+ crispants, suggesting that these specific thr isoforms function as transcription repressors for these genes, while transcription of zic and ets appear to be thraa and I-thrb+-mediated, respectively. Overall, present results show that TH signaling participates in early zebrafish development and identify $\mathrm{Tr}$ isoform-specific mediated regulation of early gene expression.

Keywords: thyroid hormone receptors, thyroid hormones, CRISPR/Cas9, development, zebrafish

\section{INTRODUCTION}

Thyroid hormones (THs) play important roles in different developmental processes and life transitional events of vertebrates (1-5). The molecular mechanisms that govern these events are complex and not fully described, but there is evidence that several of the TH-regulated effects are exerted through a genomic mechanism mediated by thyroid hormone receptors (TRs) (6), which are members of the nuclear receptor superfamily. In vertebrates, TRs are encoded by two distinct 
genes denominated thyroid hormone receptors alpha (THRA) and beta $(T H R B)$, which in turn are transcribed into several TR isoforms with tissue- and species-specific functions $(7,8)$. Aside from T3 (3,3',5-triiodo-L-thyronine), T2 (3,5-diiodo-Lthyronine) also functions as an important TR ligand known to bind preferentially to different $\mathrm{Tr}$ isoforms, at least in teleosts (9), suggesting that each of these ligands can modulate different transcriptional processes.

In a comparative scenario, zebrafish have become an invaluable tool to start unraveling some of the mechanisms involved in vertebrate developmental processes. For example, during segmentation [10-20 h post fertilization (hpf)] an anteroposterior embryo is well-defined, and somites, tail, and a rudiment of the head and eyes can be observed (10). Some transcription factors that regulate cell fate and differentiation are regulated in a fine way before segmentation starts (5-10 hpf). During gastrulation (5-10 hpf), epiboly, internalization and germinal layer formation, as well as a correct positioning of dorso-ventral, antero-posterior, and left-right axis of embryo occur $(11,12)$, and several genes involved in cell fate and organogenesis start expression during this stage. Trs are known to function as the $\mathrm{TH}$ signal modulators during zebrafish development and in concert their coding mRNA is present in the fertilized egg in high concentrations during the first $6 \mathrm{hpf}$, after which thr mRNA decreases to low or non-detectable levels until 24 hpf $(13,14)$. The fact that $t h r$ mRNAs exhibit their highest levels concomitantly with those of intra-ovum THs (15) suggests that both $\mathrm{Tr}$ and ligand are from maternal origin and that these can be functional before embryonic transcription of thr and the appearance of the thyroid gland for embryonic $\mathrm{TH}$ synthesis. $\operatorname{Tr}$ manipulation results in stronger effects than those from exogenous $\mathrm{TH}$ administration. Indeed, overexpression of $\mathrm{Tr} \alpha$ has shown dramatic effects upon craniofacial development (16), and recently, human dominant-negative TRs were employed to determine the role of these isoforms during development $(14,17)$.

The objective of the present study was to further understand the role of THs and their receptors during early development. To that end, we evaluated the effects of exogenous T3 and T2 exposure during embryogenesis as well as disrupted $t h r$ expression using the CRISPR/Cas9 methodology. Also, we analyzed the expression of some early development genes and identified if they were TH-responsive and direct thra- or thrbmediated TH targets.

\section{MATERIALS AND METHODS}

\section{Animals}

Adult zebrafish (Danio rerio) were purchased from a commercial pet store and acclimatized to laboratory conditions: flowthrough system with tap water at $28^{\circ} \mathrm{C}$ and a photoperiod 16:8 (light:dark). Embryos were obtained from natural mating, washed with tap water and cultured with E3 standard medium containing $5 \mathrm{mM} \mathrm{NaCl}, 0.17 \mathrm{mM} \mathrm{KCl}, 0.33 \mathrm{mM} \mathrm{CaCl}_{2}, 0.33 \mathrm{mM}$ $\mathrm{MgSO}_{4}$ and methylene blue. Un-injected embryos (UN) were immediately placed into an incubator at $28.5^{\circ} \mathrm{C}$. All zebrafish were maintained and handled in accordance with protocols approved by the Ethics for Research Committee of the Instituto de Neurobiología at the Universidad Nacional Autónoma de México (UNAM).

\section{Preparation of sgRNA and mRNA Cas 9}

The zebrafish thraa, thrab, and thrb gene sequences were obtained from the zebrafish information network (www.zfin.org), and the different isoforms were verified on Ensembl (www.ensembl.org).

A well-established protocol was followed for genome editing (18). The main steps are briefly described: The scoring algorithm designed and tested in zebrafish CRISPRscan [www.crisprscan. org; (19)] was used to design sgRNAs. The selected target regions and selected guides for thraa and thrab are illustrated in Supplementary Figure 2 and Table 1. In the case of the thrb gene, two guides were used: sgRNA $l$ - thrb+, which only targets the newly identified super long thrb (l-thrb+, see below), and sgRNA thrb*, which potentially targets three distinct isoforms (the well-described long (1-) $\operatorname{Tr} \beta 1$ and short (s-) $\operatorname{Tr} \beta 1$ isoforms and the newly described 1-Tr $\beta+$ ) (Supplementary Figure 3). sgRNA was synthetized by in vitro transcription using T7 Quick High Yield RNA Synthesis Kit (New England Biolabs). Cas9 mRNA was also synthetized by in vitro transcription using an XbaI-linearized pT3TSn-Cas9n plasmid as template through mMESSAGE mMACHINE T3 kit (Life Technologies). sgRNAs and mRNA Cas9 were purified by ethanol precipitation and resuspended in RNAase free water. For the CRISPR/Cas9 microinjection, Cas 9 mRNA and sgRNA mix was prepared and zebrafish embryos were injected directly with a final volume of $1 \mathrm{~nL}$ equivalent to 100 and $20 \mathrm{pg}$ of Cas9 and sgRNA per embryo, respectively.

\section{Microinjection}

Embryos for microinjections were prepared according to Rosen et al. (20). One-cell stage zebrafish embryos were injected directly with a final volume of $1 \mathrm{~nL}$ of vehicle or working solutions using $1.5 \mathrm{OD} / 1.12$ ID thinwall capillars (World Precision Instruments) and a Pneumatic PicoPump (PV 820; World Precision Instruments). To calculate intra-ovum $\mathrm{TH}$ concentration, an intra-embryonic volume of $170 \mathrm{~nL}$ was estimated. Groups of around 50 eggs were injected with the corresponding guide, either $\mathrm{TH}$ or vehicle, which consisted in DEPC water for CRISPR/Cas9 experiments and $10^{-7} \mathrm{~N}$ $\mathrm{NaOH}$ for $\mathrm{TH}$ treatments. Three independent experiments were performed per group.

\section{DNA Extraction}

A HotSHOT modified protocol (21) was used to extract larval genomic DNA, in which $45 \mu \mathrm{L}$ of $50 \mathrm{mM} \mathrm{NaOH}$ were added to each individual larva, followed by a $30 \mathrm{~min}$ incubation at $95^{\circ} \mathrm{C}$. The samples were cooled at $4^{\circ} \mathrm{C}$, and $5 \mu \mathrm{L}$ of $1 \mathrm{M}$ Tris- $\mathrm{HCl}$, pH 8.0 were added to neutralize the solution. The samples were centrifuged to pellet debris and $5 \mu \mathrm{L}$ of the supernatant were used for $50 \mu \mathrm{L}$ PCR reactions.

\section{Crispant Verification}

To verify that the zebrafish larvae contained the desired gene mutations, a fragment of thraa, thrab, and thrb genes that included the CRISPR/Cas9 target sites was amplified. To this end, 
TABLE 1 | Primers and templates.

\begin{tabular}{|c|c|c|c|c|c|}
\hline Gene target & Sequence identifiers & Forward primer $\left(5^{\prime}-3^{\prime}\right)$ & Reverse primer $\left(5^{\prime}-3^{\prime}\right)$ & Primer position & Length (bp) \\
\hline \multicolumn{6}{|c|}{ REAL TIME PCR PRIMERS } \\
\hline dio2 & NM_212789.4 & GCAGCGCATGTTAACCACAG & GTTGTGGGTCTTACCGCTGA & Exon 1-2 & 160 \\
\hline dio3a & NM_001256003.1 & CGCTCGTGTGTCTGCTCATT & CAGAGACTCCCAGCTGAACA & Exon 1 & 175 \\
\hline thraa & NM_131396.1 & ATGGAAAACACAGAGCAGGAG & AGGAACAGAGATGCTCTTGTC & Exon 2-4 & 132 \\
\hline thrab & ENSDART00000153187.2 & GGATGGAAATAAGGTGAATGGAAC & GGTAGTGATATCCGGTAGCTITG & Exon 3-5 & 210 \\
\hline s-thrb1 & NM_131340.1 & AGAAGACTGTATGGGATCGAC & GTCTTCTGGCAGGAATITGCG & Exon 9-10 & 134 \\
\hline I-thrb1 & ENSDART00000151766.3 & AGAAGACTGTATGGGATCGAC & GGCTTGGCTTCCTTCACCC & Exon 7-8 & 154 \\
\hline mct8 & NM_001258230 & GTTCGGGAAGATCGGAGACC & AACACGGCACACTGAGGAAT & Exon 21-22 & 111 \\
\hline ets1 & NM_001017558.1 & GAGATITCTGGACCTGGCAC & GAAATATTCGGAGGGATAGCGG & Exon 4-5 & 145 \\
\hline eve1 & NM_131114.1 & GGGAACAGCTGACTCGTCTC & TGTCCTTCATTCTCCGGTTC & Exon 2-3 & 141 \\
\hline$f g f 1 b$ & NM_001105278 & CATGAGACTGGACTATACCTTGC & GTCCTGATATCTCTGCGAACG & Exon 2-3 & 138 \\
\hline foxd3 & NM_131290 & GTCCCGTCAAATATCATCTCCG & GCCTATAGTTCGTGCTGTATCG & Exon 2-3 & 150 \\
\hline$m s \times 1 a$ & NM_131273.1 & TCACACCCGTITCACAGAC & CGGCAAACTTCACAAGTCAC & Exon 2-3 & 147 \\
\hline sox10 & NM_131875.1 & TCAATATCCGCACCTGCAC & CGCTTATCCGTCTCGTTCAG & Exon 2-3 & 82 \\
\hline pax7a & ENSDART00000172008.3 & TCTGCAAAGTTCCTCCGGATT & CTGCAGTGCACAATGCCAAA & Exon 1 & 310 \\
\hline zic1 & NM_130933.2 & CTACACACATCCCAGTTCTCTC & TCTGGTITCTGTGGAAGGG & Exon 2-3 & 143 \\
\hline mbpa & ENSDART00000052556.8 & GAGGAGACAAGAAGAGAAAGGG & GAAATGCACGACAGGGTTG & Exon 1-2 & 83 \\
\hline$m p z$ & NM_194361.2 & ACCTGTGATGCCAAGAACC & TTGCCACAACGAGGATCA & Exon 3-4 & 148 \\
\hline olig2 & ENSDART00000060006.5 & CGAGTGAACTGGAATAGCCTTAC & GCTCGTGTCAGAGTCCATG & Exon1-2 & 134 \\
\hline$p / p$ & ENSDART00000003514.8 & ACACTGTTAACGTCCTGTCAG & CTGGTGCTITGCATATGTTGG & Exon 4-5 & 147 \\
\hline Ism12b & NM_213148.1 & AGTTGTCCCAAGCCTATGCAATCAG & CCACTCAGGAGGATAAAGACGAGTC & Exon 3-4 & 300 \\
\hline
\end{tabular}

\begin{tabular}{|c|c|c|c|}
\hline Gene target & Sequence identifiers & Forward primer $\left(5^{\prime}-3^{\prime}\right)$ & Reverse primer $\left(5^{\prime}-3^{\prime}\right)$ \\
\hline \multicolumn{4}{|c|}{ gDNA PRIMERS } \\
\hline thraa & ENSDARG00000000151 & TGTCAGATGGCCAAATGGAGT & CTGGTTGCGGGTGATITTGT \\
\hline thrab & ENSDARG00000052654 & AGCTCTCGGAGCTGAAAGTG & ACCAGTGTAAGGAATAAAGTTGCT \\
\hline thrb (1-thrb+) & ENSDARG00000021163 & GACATAGCCCATGGTGTAAG & CTITCTTATGTGGCCCTTGC \\
\hline thrb (thrb*) & ENSDARG00000021163 & GCATGGCTACAGACTGTAAG & GTTGTCAACAGGGAAGAGAC \\
\hline
\end{tabular}

Templates for in vitro transcription of sgRNAs

\begin{tabular}{ll}
\hline thraa & taatacgactcactataGGAGCGGTAATGATAGCCAGgttttagagctagaa \\
thrab & taatacgactcactataGGGAAAGAACAGCCAGTGTTgttttagagctagaa \\
l-thrb+ & taatacgactcactataGGGTGAGTTATGCACCATGGgttttagagctagaa \\
thrb* & taatacgactcactataGGGAGAACCGTGAACGCCGAgttttagagctagaa \\
Generic for template assembly & AAAAGCACCGACTCGGTGCCACTITCAAGTTGATAACGGACTAGCCTTATITaacttgctatttctagctctaaaac
\end{tabular}

a pair of primers for each gene was designed (see Table 1). An equal volume of DNA from 8 larvae of the same experimental condition were mixed (un-injected wild type and injected) for PCR amplification using Platinum Taq DNA Polymerase (Invitrogen) and subsequently column-purified (DNA Clean \& Concentrator $^{\mathrm{TM}}$; Zymo Research). Purified PCR amplicons were either directly sequenced or used for subcloning in the TA vector pGEM-T Easy (Promega) to analyze the mutant allele populations from the injected larvae. Isolated colonies resulting from competent bacteria transformations were plasmid extracted and sent to sequencing using the universal primer $\mathrm{T} 7$.

\section{Quantitative PCR}

The expression of selected genes was quantified in native, THtreated and crispant larvae. To that end, total RNA was extracted from 0 un-injected zebrafish fertilized eggs and 9 hpf embryo pools (8-16) with Trizol Reagent (Life technologies). RNA was reverse transcribed with RevertAid Reverse Transcriptase (Thermo Scientific) from $1 \mu \mathrm{g}$ of total RNA and $0.5 \mu \mathrm{g}$ oligo (dT). Specific oligonucleotides were designed with Realtime PCR tool IDT. PCR products were obtained using a proofreading DNA polymerase for $10^{\prime}$ at $95^{\circ} \mathrm{C}, 10^{\prime \prime}$ at $95^{\circ} \mathrm{C}$, $10^{\prime \prime}$ at $61^{\circ} \mathrm{C}$ and $10^{\prime \prime}$ at $72^{\circ} \mathrm{C}$ for 40 cycles and were cloned into pJET1.2/blunt vector (Thermo Scientific). Constructs were verified by sequencing, and standard curves that ranged from $10^{5}$ to $10^{9}$ molecules/ $\mu \mathrm{L}$ were prepared. In all cases, reactions contained $1 \mu \mathrm{L}$ of reverse transcribed reaction, $6 \mu \mathrm{L}$ Maxima SYBR Green/ROX qPCR Master Mix (Thermo Scientific) and 250 or $500 \mathrm{nM}$ forward and reverse oligonucleotides in a final volume of $12 \mu \mathrm{L}$. A Step One instrument was used for detection and data 


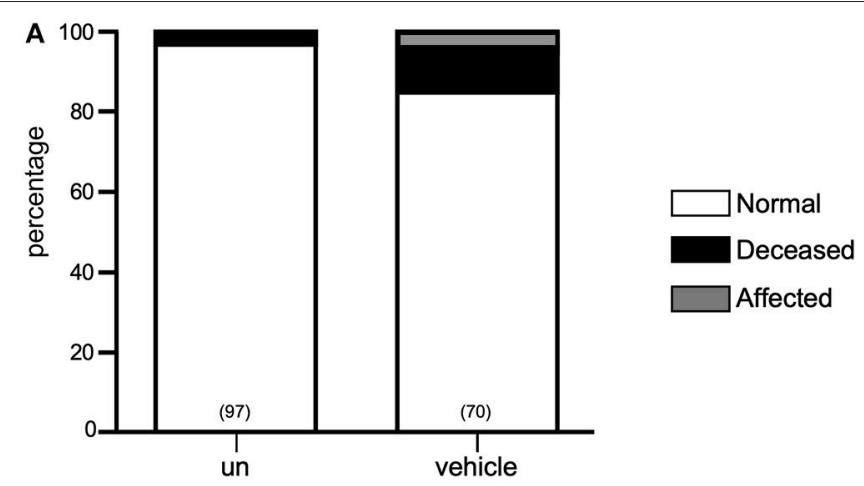

B

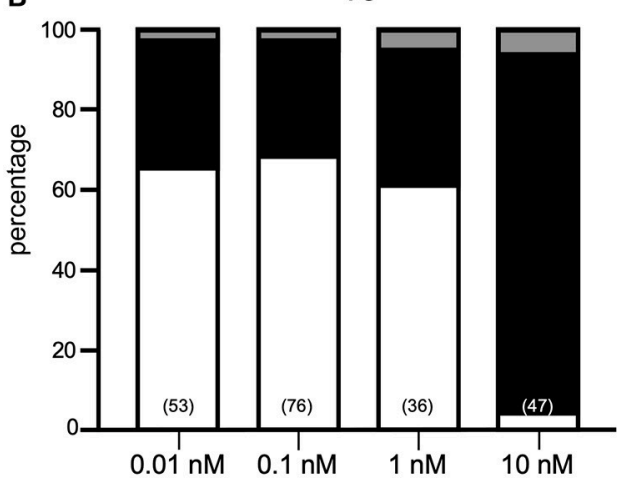

C

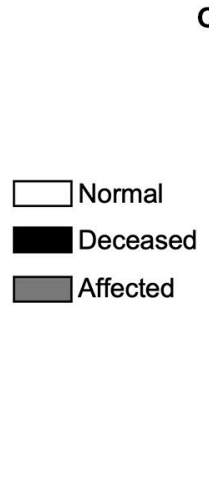

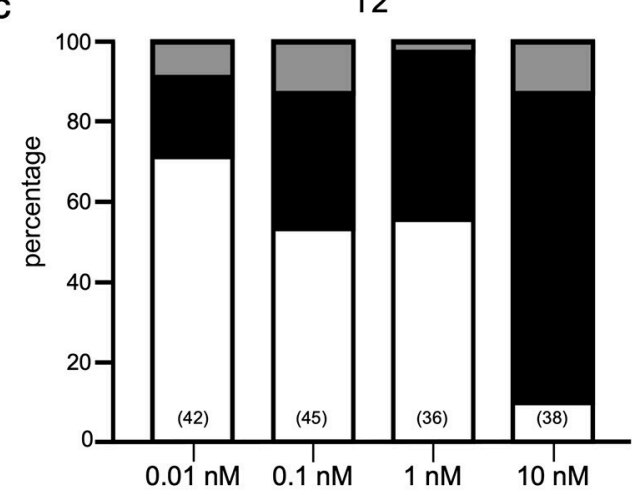

FIGURE 1 | Effect of THs in zebrafish development. (A) Percentage of normal, affected and deceased larvae in un-injected (UN), vehicle or TH treatments. Neither T3 (B) nor T2 (C) had effects upon morphology at $0.1-10 \mathrm{nM}$ but is toxic at $10 \mathrm{nM}$.

analysis according to the manufacturer's instructions (Applied Biosystems). The absolute mRNA concentration was expressed as molecules per microgram of total mRNA used in RT reaction and obtained by interpolation with the standard curve and normalized with reference gene lsm $12 \mathrm{~b}$ (22) in each experimental sample. Oligonucleotides used for gene quantifications are listed in Table 1.

\section{Statistical Analysis}

Results were analyzed using ANOVA coupled to a Tukey post-hoc test (control vs. treatments) and GraphPad Prism 7. Differences were considered statistically significant at $P \leq 0.05$.

\section{RESULTS}

\section{Effects of Exogenous T3 and T2 Exposure During Embryogenesis}

Until now, only immersion administration protocols have been used to deliver exogenous $\mathrm{TH}$ into the teleost embryo (23, 24). For this study we microinjected one-cell stage zebrafish embryos with $\mathrm{T} 3$ or T2 at concentrations ranging from 0.01 , $0.1,1$, and $10 \mathrm{nM}$ and observed for effects on the general body plan or on mortality during the first $48-50 \mathrm{hpf}$. As depicted in Figure 1, $19-41 \%$ and $30-33 \%$ of mortality was observed after the injection of $0.01,0.1$, and $1 \mathrm{nM}$ of both $\mathrm{T} 3$ and T2, respectively, however, mortality increased to $76-89 \%$ when embryos were injected with $10 \mathrm{nM}$ of either hormone, clearly showing toxic effects. In all cases, the percentage of affected embryos was similar to those vehicle-injected (below $6 \%$ for T3 and $13 \%$ for $\mathrm{T} 2$ ), suggesting that the microinjection did not influence development, as well as that the early exposure to the hormone alone was not sufficient to elicit effects at least upon the phenotype of the embryo (Figure 1). Since transactivating assays showed that zebrafish TRs were activated with $0.1 \mathrm{nM}$ (Supplementary Figure 1), and a slightly lower mortality rate was observed with this concentration compared to 1 and $10 \mathrm{nM}$, subsequent experiments were performed with $0.1 \mathrm{nM}$ of either T3 or T2.

\section{thraa but Not thrab Impacts Early Zebrafish Development}

Two thra genes have been described in teleosts, but from these genes, only thraa has been more extensively studied. In zebrafish, two thraa transcripts have been described, which differ in the presence of an extension of around 12 amino acids in the C-terminal domain (Figure 2). In contrast, only one transcript has been described for thrab, however, we identified two transcripts in Ensembl that differ by 6 amino acids at the N-terminal of the protein (Figure 2). As mentioned above, 


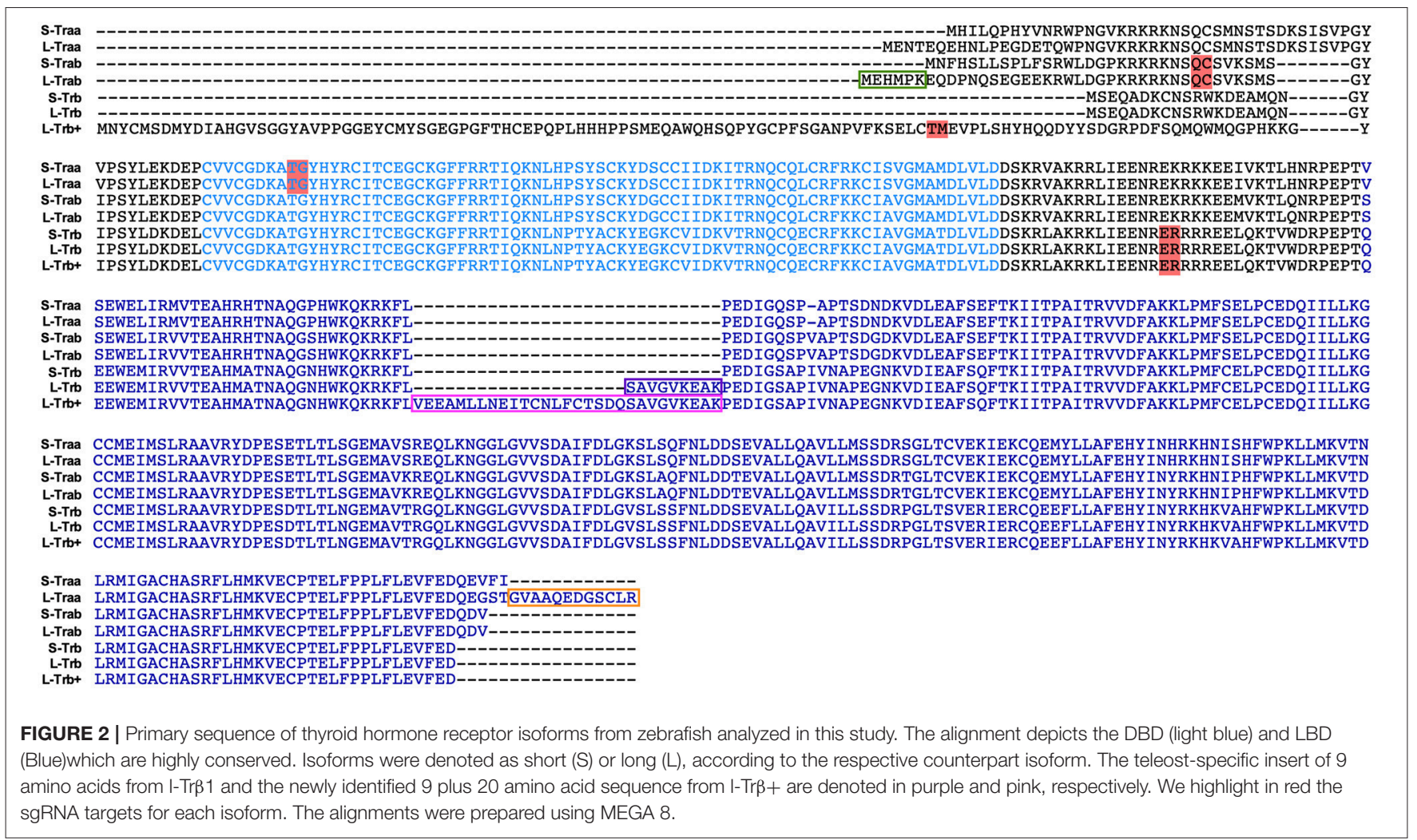

for this study, guides to disrupt thra genes were designed to target the two identified isoforms per gene. Designed sgRNA guides were effective to produce a variety of mutations reflected in the electropherograms from crispant DNA with respect to that from wildtype embryos (Supplementary Figures 2, 3). Our results show that approximately $35 \%$ of the thraa crispant larvae presented a clear loss of symmetry and laterality (asymmetric size and position of external morphology mainly of eyes, head, and tail) observed as early as $24 \mathrm{hpf}$ when the effects were severe, or from 3 to 4 days post-fertilization in mildly to moderately affected larvae when the body plan had taken form (Figure 3A; Supplementary Figure 4). Moreover, this group of crispants exhibited a $30 \%$ mortality (Figure 3B). In contrast, when thrab crispants were analyzed, no effect was observed in larvae (Figures 4A,B), and mortality was only 5.5\%. Altogether, these results strongly suggest a novel role for thraa in the body plan development program, whereas thrab, although expressed in early development $(13,25)$, does not seem to participate in these processes.

\section{Zebrafish L-trb+ Crispants Show Impaired Embryogenesis}

As mentioned, several functional teleost-specific thrb isoforms have been identified. Of these, the most representative are two gene products that contain or not an insertion of nine amino acids in the ligand binding domain (LBD) of the protein and that have been referred to as long or short thyroid hormone receptor $\beta 1$ (S- or L- $\operatorname{Tr} \beta 1$ ). However, some metamorphic species have been shown to express an additional thrb isoform that contains 20 amino acids adjacent to the 9 -amino acid insert, and that has been denominated L- $\operatorname{Tr} \beta 1+(8)$. Interestingly, we identified (Ensembl) an isoform that had not been previously identified in zebrafish that contains a 111 amino acid N-terminal fragment, similar in length to that of the human $\operatorname{Tr} \beta 2$, but with only $26 \%$ of conserved amino acids within this region (Figure 2). The low sequence identity in the fragment raises the doubt that the isoform could indeed be a $\operatorname{Tr} \beta 2$. Furthermore, this $\operatorname{Tr} \beta$ isoform contains the 29-amino acid insert, as described for the metamorphic species $(\mathrm{L}-\operatorname{Tr} \beta 1+)$. Given the ambiguity to clearly identify this isoform in terms of sequence identity, in this study we have denominated it $\mathrm{L}-\operatorname{Tr} \beta+$.

To unravel the putative role of this as well as the other two $\operatorname{tr} \beta 1$ during early development, crispants for the three isoforms were generated by using the guide $t h r b^{*}$, as well as crispants that were directly targeted to disrupt l-trb+. Surprisingly, only crispants specifically generated to disrupt $l$ trb + presented effects in 30\% of larvae, showing a loss of symmetry and laterality in the same manner as thraa crispants (Figures 3A,C). In contrast, crispants resulting from a target site that disrupts a shared $\operatorname{Tr} \beta 1$ sequence (Figure 2) only showed minor effects as when using the guide that disrupts $l$-trb + alone (Figures 4A,C). Although intriguing, these results evidence a clear functional role of the novel l-trb+ isoform at least during early development. No morphological defects were observed in $60 \%$ of crispants generated with sgRNA thrb*, an observation that could result from the nature of the sgRNA target site, which renders transcripts that still contain the DNA-binding domain (DBD), possibly allowing the truncated protein to bind 
A

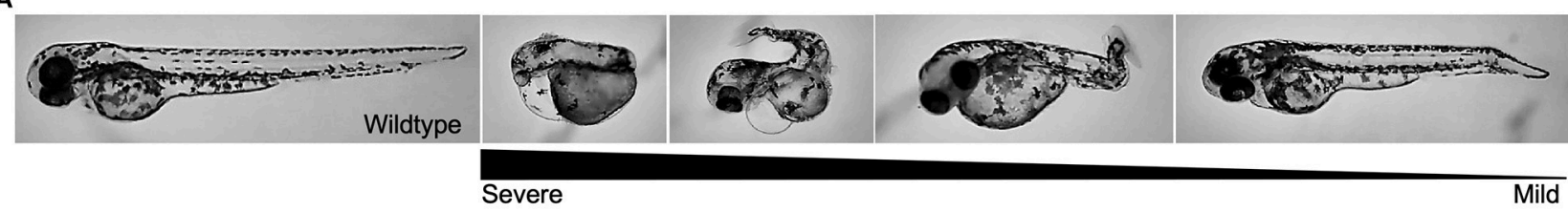

B

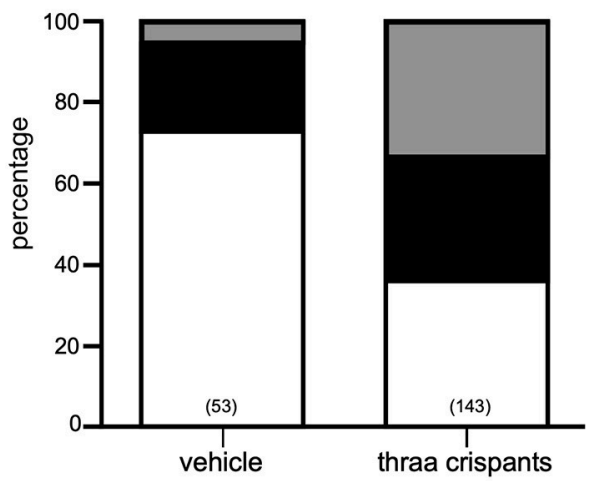

D

WT TGTGCGGAGACAAGGCCACTGGCTATCATTACCGCTGCATCACATGTGAGGGC -2pb TGTGCGGAGACAAGGCCAC-G-CTATCATTACCGCTGCATCACATGTGAGGGC -2pb TGTGCGGAGACAAGGCCACTG-C-ATCATTACCGCTGCATCACATGTGAGGGC -3pb TGTGCGGAGACAAGGCCACT---TATCATTACCGCTGCATCACATGTGAGGGC $-2 /+3 p b$ TGTGCGGAGACAAGGCCACTGCATTATCATTACCGCTGCATCACATGTGAGGG
C

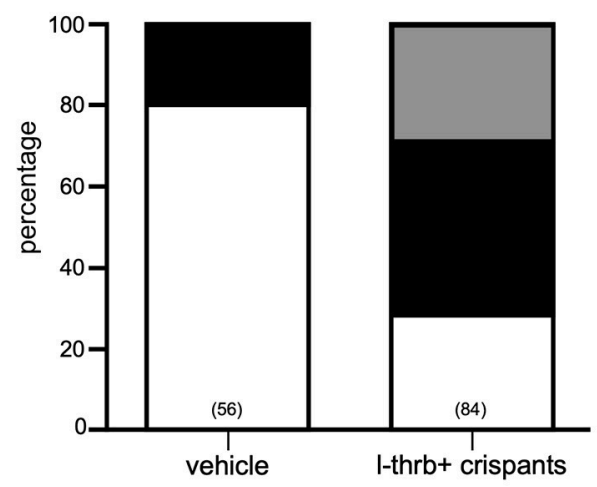

thro

WT CTAACCCTGTTTTCAAGAGTGAGTTATGCACCATGGAGGTGCCTCTCAGTCAC -2pb CTAACCCTGTTTTCAAGAGTGAGTTATGCAC--TGGAGGTGCCTCTCAGTCAC -3pb CTAACCCTGTTTTCAAGAGTGAGTTATG---CATGGAGGTGCCTCTCAGTCAC -7pb CTAACCCTGTTTTCAAGAGTGAGTT-------ATGGAGGTGCCTCTCAGTCAC -19pb CTAACCCTGTTTTCAAG--

FIGURE 3 | Characterization of thraa and l-thrb+ crispants. (A) thraa and l-thrb+ crispants show diverse phenotypes with several degrees of body plan affectations as compared with wild-type at $3 \mathrm{dpf}$. Various phenotypes were observed due the mosaicism of the mutation. (B,C) Represent the percentage of normal, affected and deceased larvae after sgRNA and Cas9 treatment. (D) DNA sequence of sgRNA target site for CRISPR/Cas9 is denoted in gray, whereas PAM sequence is denoted in blue. Insertion-deletions (indels) are highlighted in yellow.

to TH-responsive elements in target gene promoters and repress gene transcription. However, the $32 \%$ mortality observed in this group could also correspond to the population where $l$ $t r b+$ expression was most affected, further suggesting that the canonical thrb isoforms do not appear to be involved in early developmental events.

\section{Changes in Development-Related Genes at $9 \mathrm{hpf}$}

Both thraa and $l$-thrb + crispants showed a clear loss of symmetry and laterality, visualized at $24 \mathrm{hpf}$ when a well-defined anteroposterior pattern was observed in control larvae. As previously mentioned, critical events that determine cell fate for these developmental stages, like epiboly, internalization and germinal layer formation, start in the gastrula (approximately 5-10 hpf), $(10,26)$. Thus, we hypothesized that some genes that are expressed during early development could indeed be TH targets, with Tr-specific signaling pathways. To prove our hypothesis, we chose $9 \mathrm{hpf}$ embryos, which were at the onset of segmentation, to analyze the expression of sets of genes known to be part of TH signaling (dio2, dio3, mct8, thraa, thrab, s-thrb1, l-thrb1), and genes involved in symmetry and laterality (eve, fgf, zic, pax 7, msx, foxd3, sox 10, ets) and in myelination ( $m p z, m b p$, olig 2, plp1b) (Table 1). Since myelination does not start until 48 hpf in zebrafish (27), the latter set of genes was included as a negative control group. This screen was performed in fertilized un-injected eggs to determine gene expression at time zero and in the 9 hpf vehicle-injected embryos or embryos treated with T3 and T2, as well as in crispants generated for all thr isoforms. As $9 \mathrm{hpf}$ is too early to detect body plan malformations, the mRNA pool samples of crispants were heterogeneous since we were unable to distinguish between affected and normal embryos. Nonetheless, we were still able to detect clear changes in mRNA expression in the different experimental groups, with clear statistical significance compared to controls. Exogenous TH exposure influenced the regulation of several genes at this stage of development, showing that the expression of TH-responsive genes is receptive to $\mathrm{TH}$ regulation during gastrulation. The fact that T2 had an effect upon gene regulation suggests that in this developmental stage, as in the juvenile and adult stages, T2 is a relevant TR alternative ligand $(28,29)$ (Lazcano et al. under review) $)^{1}$.

As observed in Figures 5-7, mRNA from dio2, thraa, s-thrb1, l-thrb1, $m b p$, ets, and $f g f$ was highly expressed at the stage of one-cell embryo (0 hpf), while mRNA expression of $m c t 8, m p z$, eve, foxd $3, m s x$, and $z i c$ was not detected, showing the maternal origin of some transcripts. Indeed, transcripts from maternal origin are present in the oocyte, and zygotic transcription starts around 2 to $3 \mathrm{hpf}$. Other transcripts ( $m c t 8$, eve, foxd3, $m s x$, and $m p z$ ) were only detected after $9 \mathrm{hpf}$, evidencing onset of zygotic

${ }^{1}$ Lazcano I, Hernández-Puga AG, Orozco A. Alternative ligands for thyroid hormone receptors. A molecular perspective. Mol Cell Endocrinol. 
A

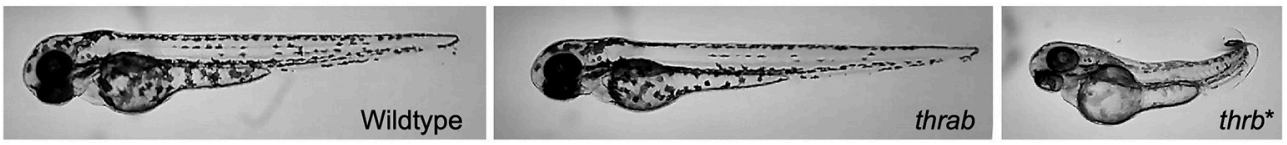

B

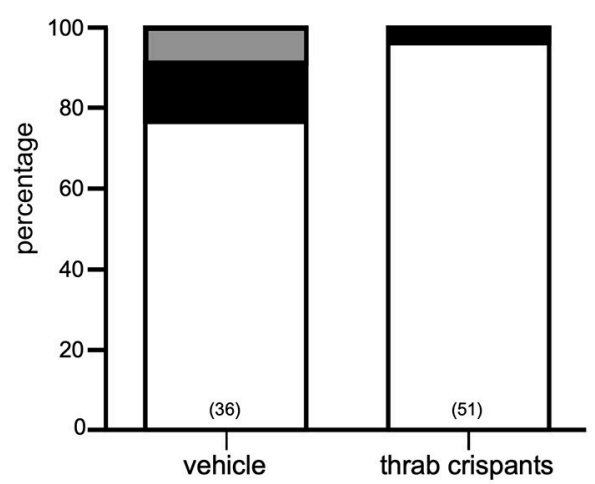

D thrab

WT GATGGCCCGAAAAGGGAAGAGAAAGAACAGCCAGTGTTCCGTGAAGAGCATGT -2pb GATGGCCCGAAAAGGGAAGAGAAAGAACAGCCA--GTTCCGTGAAGAGCATGT

+1pb ATGGCCCGAAAAGGGAAGAGAAAGAACAGCCAGTTGTTCCGTGAAGAGCATGT $-1 /+9 \mathrm{pb}$ AAAAGGGAAGAGAAAGAACAGCCAGTTGGGCAGTGCTTCCGTGAAGAGCATGT $-3 /+12 \mathrm{pb}$ AAAAGGGAAGAGAAAGAACAGCCGGTGAAAGAACAGTTCCGTGAAGAGCATGT
C

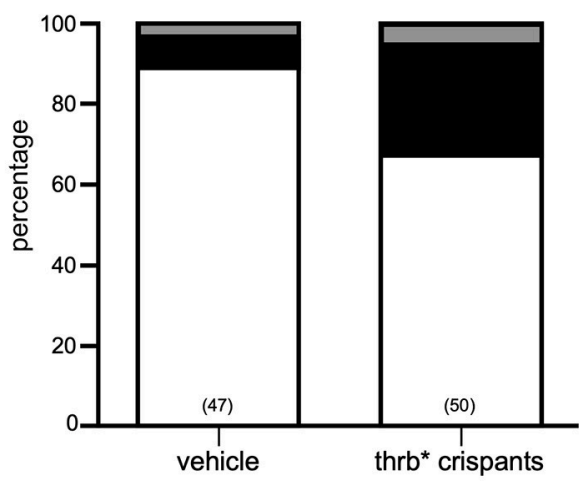

thrt

WT AAGCGGAAGCTGATTGAGGAGAACCGTGAACGCCGACGGCGTGAGGAGCTGCA -4pb AAGCGGAAGCTGATTGAGGAGAACCGTGAACG---CGGCGTGAGGAGCTGCA -7pb AAGCGGAAGCTGATTGAGGAGAACCGTGA------CGGCGTGAGGAGCTGCA -75b AACGGAAGCLGATGAGGAGAACGT

FIGURE 4 | Characterization of thrab and I-thrb* crispants. (A) thrab crispants do not show effects upon zebrafish morphology, whereas l-thrb* crispants generated with an sgRNA able to target all thrb isoforms showed a low percentage of affected phenotypes characterized by laterality. (B,C) Represent the percentage of normal, affected and deceased larvae after sgRNA and Cas9 treatment. (D) The DNA sequence of sgRNA target site for CRISPR/Cas9 is denoted in gray, whereas PAM sequence is denoted in blue. Insertion-deletions (indels) are highlighted in yellow.

gene transcription (10). Furthermore, we identified genes that were up- (thraa, thrab, msx) or down-regulated (dio2, eve, pax 7) by both T3 and T2 at $9 \mathrm{hpf}$, as compared with vehicle-injected embryos, and genes that were specifically up-regulated by T3 (sox10) or by T2 (mct8, olig2, zic), as well as genes down-regulated by $\mathrm{T} 2$ (foxd3). We also identified genes that did not respond to TH treatment (dio3, mbp, mpz, plp, ets, fgf, pax7). Thus, out of the three sets of genes, at least those related to TH signaling and early development were indeed TH-responsive. The early exposure of $\mathrm{T} 2$ resulted in an up-regulation of olig2, a gene associated to myelination, suggesting a direct regulatory effect of this hormone. As an attempt to identify if the $\mathrm{TH}$ response was mediated by a specific thr, we analyzed the expression of the three sets of genes in thr crispants. Only sox10, eve and zic specifically increased their expression in thraa crispants, sox10, ets and eve in l-thrb + crispants and $m b p$ in thrab, suggesting that for these genes, thraa, l-thrb+, and thrab respectively, function as repressors of transcription. thrb* crispants, where all thrb isoforms are targeted, exhibited an increased expression of dio3, eve, pax7, and zic, suggesting that the regulation of eve is mediated by $l$-thrb + and that of the other genes by a different thrb isoform. In contrast, we observed a more diverse response on gene expression for thrab crispants: the expression of $m b p$ and sox10 was discretely increased, while that of dio2, ets, and eve decreased, suggesting that thrab could have other roles in later stages of development.

\section{DISCUSSION}

This is the first study in which thr crispants were analyzed in order to further understand the role of Trs in early development. We found that larvae from thraa and $l$-thrb + crispants presented profound deleterious effects upon symmetry and laterality, suggesting early novel roles of these $\mathrm{Tr}$ isoforms in the body plan developmental program. We also explored the expression of early development genes known to be involved in symmetry and laterality in $t h r$ crispants and identified direct thra- or thrbmediated $\mathrm{TH}$ targets.

Early TR expression has been described before gastrula in teleosts and birds $(14,30)$ and during the first trimester of gestation in the human fetal brain (31). As in the present study, THRA is the most representative TR expressed gene (14, 31 ), suggesting that $\mathrm{TR} \alpha$-mediated $\mathrm{TH}$ signaling regulates early developmental events in all vertebrates. It is thought that THs do not participate in early vertebrate development, only after neural tube closure $(32,33)$, however, and irrespective of the vertebrate species, the embryo is always exposed to low $\mathrm{TH}$ levels from maternal origin, and TR mRNA is present from the onset of development $(13,14)$. Studies aimed to analyze early ontogenetic TH effects in zebrafish have shown that these hormones accelerate hatching and pigmentation (23). In the present work we did not detect any of these effects with our administration protocol (data not shown), but we did observe 

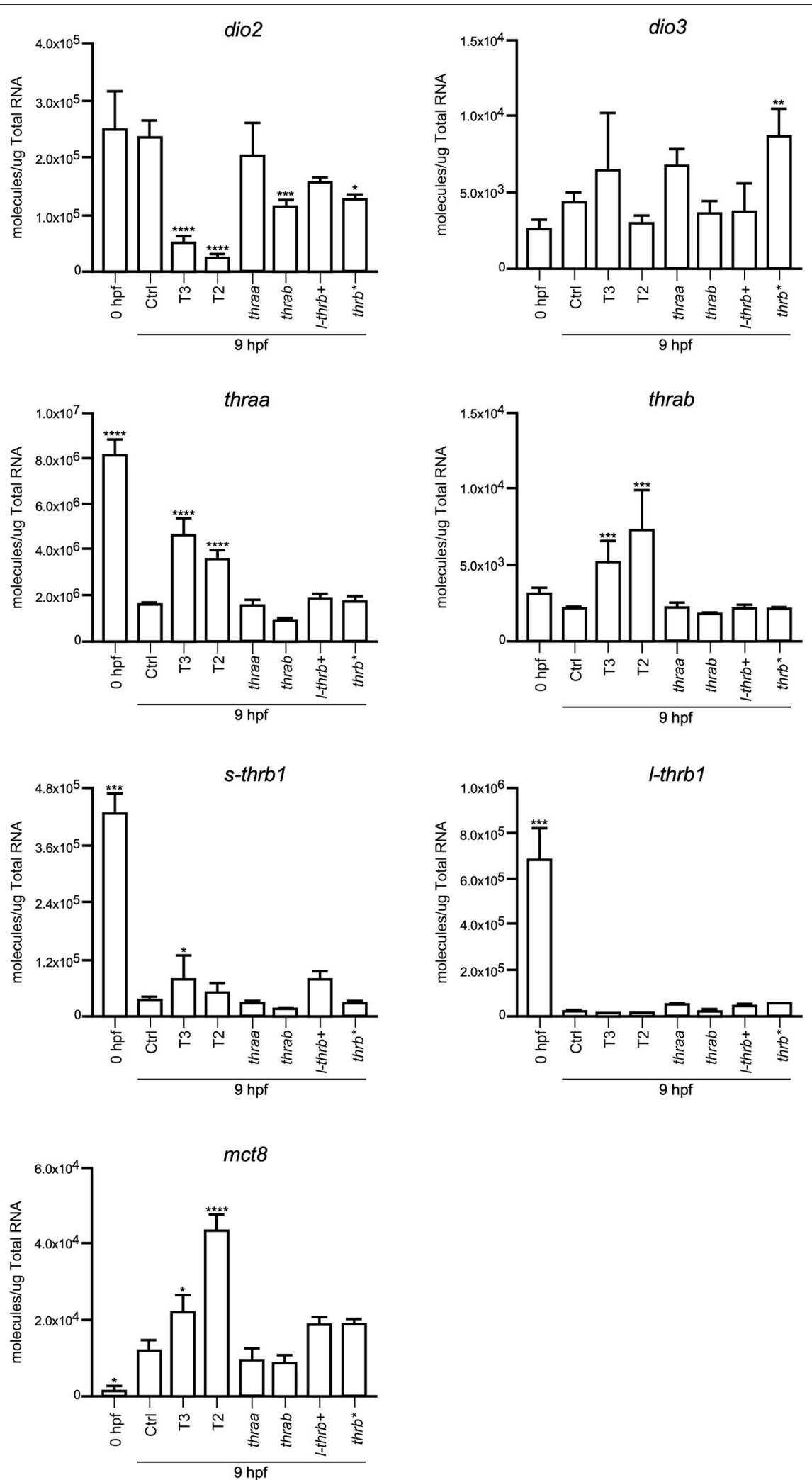

FIGURE 5 | Embryonic mRNA expression of TH-signaling genes dio2, dio3, thra, I-thrb1, s-thrb1, and mct8. Total RNA was extracted from 9 hpf zebrafish embryo pools (8-16) for qPCR. The plots show mean values \pm SEM results of three independent biological experiments. Statistical analysis was performed using one-way ANOVA coupled with a Tukey's multiple comparisons test with respect to control groups. Significance is indicated ${ }^{\star} p<0.05$, ${ }^{\star \star} p<0.01,{ }^{\star \star \star} p<0.005,{ }^{\star \star \star \star} p<0.001$. 

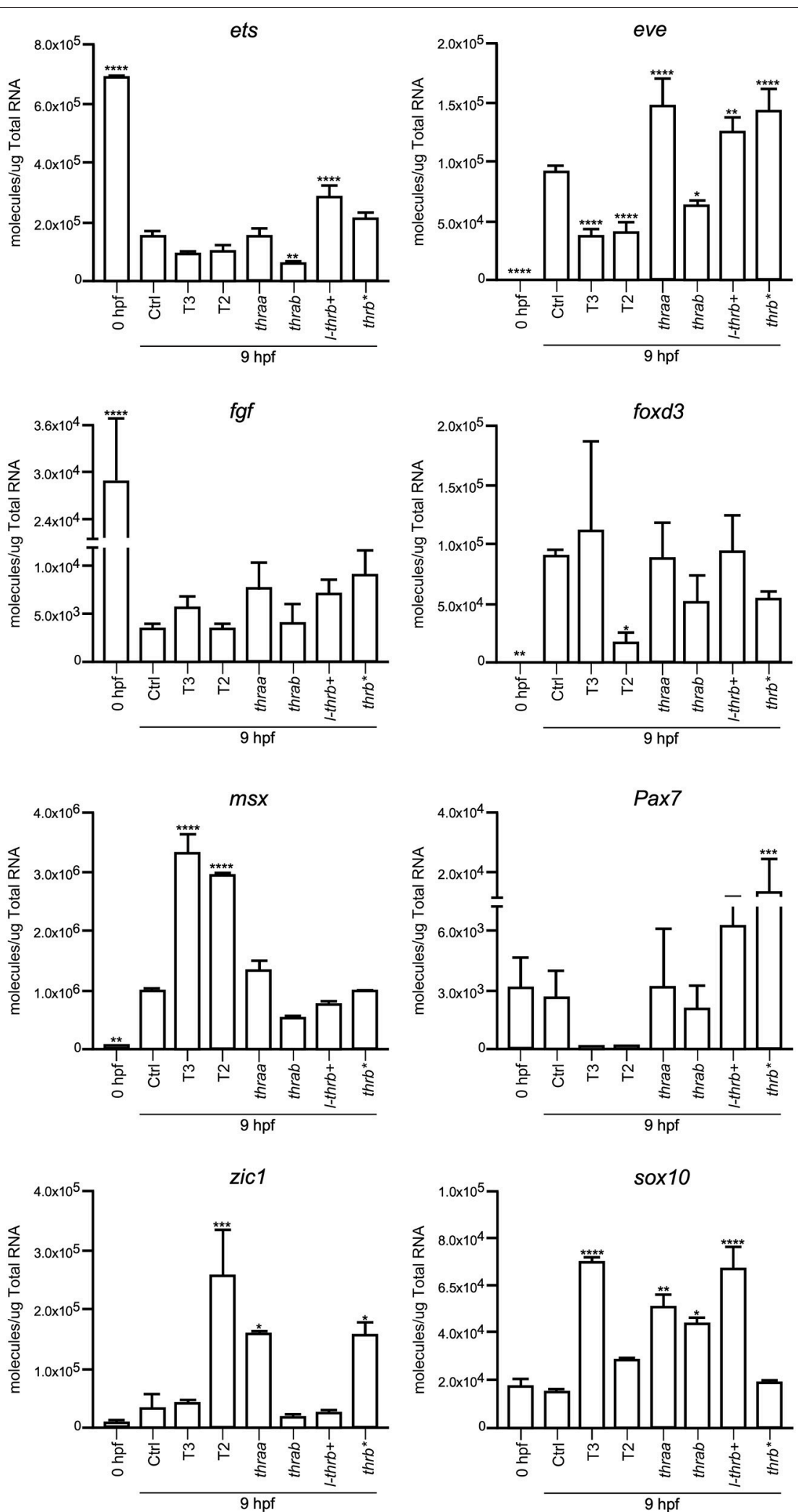

FIGURE 6 | Embryonic mRNA expression of early development genes eve, fgf, zic, pax7, msx, foxd3, sox10, and ets. Total RNA was extracted from 9 hpf zebrafish embryo pools (8-16) for qPCR. The plots show mean values \pm SEM results of three independent biological experiments. Statistical analysis was performed using one-way ANOVA coupled with a Tukey's multiple comparisons test with respect to control groups. Significance is indicated ${ }^{\star} p<0.05$, ${ }^{\star \star} p<0.01$, ${ }^{\star \star \star} p<0.005$,

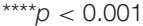



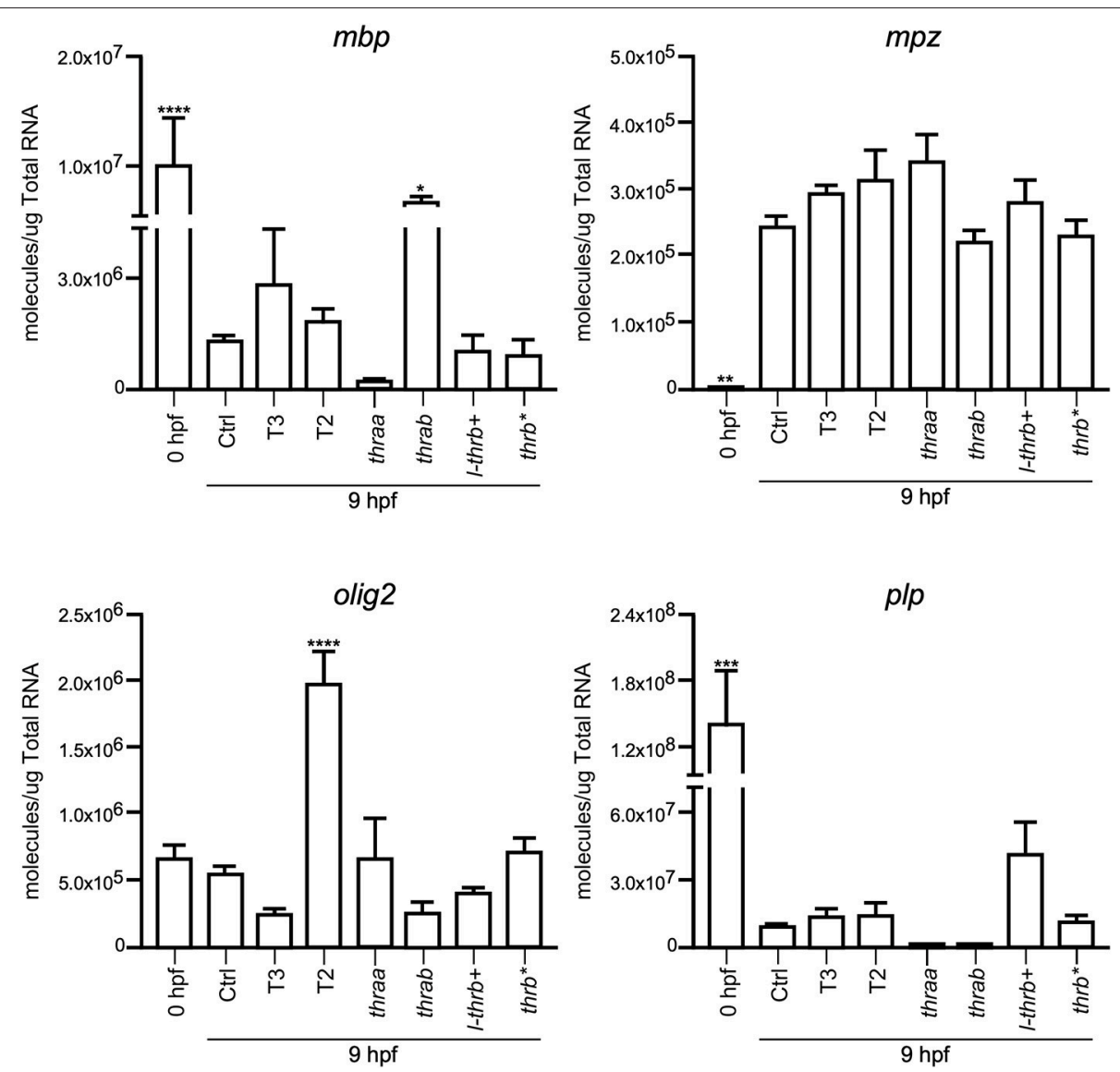

FIGURE 7 | Embryonic mRNA expression of genes involved in myelination (mpz, mbp, olig2, plp1b). Total RNA was extracted from 9 hpf zebrafish embryo pools (8-16) for qPCR. The plots show mean values \pm SEM results of three independent biological experiments. Statistical analysis was performed using one-way ANOVA coupled with a Tukey's multiple comparisons test with respect to control groups. Significance is indicated ${ }^{\star} p<0.05,{ }^{* \star} p<0.01,{ }^{\star \star *} p<0.005$, ${ }^{\star \star \star *} p<0.001$.

changes in the transcription of several genes associated with $\mathrm{TH}$ signaling and to body symmetry. Indeed, dio2, thraa, s-thrb1, and l-thrb1 were found to be highly expressed in one-cell embryos, emphasizing mRNA of maternal origin and the concomitant early onset of TH signaling. Furthermore, the expression of genes associated with body symmetry like eve, foxd $3, m s x$, and $z i c 1$, was undetectable at the one-cell stage, but detectable in $9 \mathrm{hpf}$ embryos evidencing the time-specific windows of gene expression during development. The fact that $\mathrm{TH}$ regulated some of these genes as early as $9 \mathrm{hpf}$ clearly suggests that maternal THs regulate the transcription of genes involved in their own transport, metabolism and signaling, as well as the onset of cellular and molecular mechanisms involved in body symmetry and laterality. Given these observations, it was puzzling that no clear effects upon larval phenotype were evident after TH treatment. One possible explanation is that the fine down-regulatory effect that THs exert upon dio2 expression (Figure 5) could serve as a protective mechanism from $\mathrm{TH}$ excess. Other possible explanations could involve particular TR action mechanisms yet to be elucidated.

The study of TRs and their ligands during development has focused on the central nervous system, where it is known that they are required to perform certain actions mainly related to neurogenesis and myelination (33-36) and other possible roles may have been overlooked. The zebrafish model has recently been used to address some relevant aspects of TR-TH function during development. Since teleosts underwent a specific round of genomic duplication, their genome contains several copies of genes that are absent in other taxa (37). In the case of zebrafish thr, the presence of a second copy of the thraa gene has been demonstrated and it has been called thrab $(13,25)$. The alignment of the primary sequence of the isoforms resulting from both thra genes shows differences only at the N- and Cterminal of the protein (Figure 2), while the DBD and the LBD show a high degree of identity, suggesting that all isoforms generated from the thraa and thrab genes could bind to DNA and ligate THs. In this study, the sgRNA designed to disrupt each gene modifies the open reading frame at the beginning of transcription, prior to the $\mathrm{DBD}$, in both cases affecting the synthesis of all the possible isoforms for thraa and thrab that we identified by Ensembl. The resultant crispants carry the induced mutation, but the grade of penetrance is undefined because of the nature of the changes in the nucleotide sequence (monoallelic, biallelic in- or out of frame, in different cell types or occur over different time frames). Due to this crispant nature, and as determined by sequencing, we identified a great variety of 
mutated alleles in all four target genes analyzed (Figures 3D, 4D), most of them resulting in premature stop codons and frameshifts. This variety of alleles is reflected in the obtained phenotypic diversity (Supplementary Figure 4), however, only thraa crispants presented severely affected larvae. Indeed, thraa crispants showed high mortality, clear malformations, asymmetry and altered laterality, while thrab crispants did not. It is possible that thrab mutations could affect development in a different way, without evident abnormalities in body plan, whereas thraa could regulate the transcription of body plan genes during early development (see below). However, studies to corroborate the biological activity of the receptors encoded by thrab, as well as some possible roles during other stages of the zebrafish life cycle must be tested experimentally.

Although only a single copy of the thrb gene has been identified with the exception of eels $(38,39)$ and Xenopus laevis (40) which contain two copies of thrb, this gene has other characteristics that could confer biological plasticity, like the presence of several isoforms that differ in the N-terminal and/or the LBD (Figure 2). Zebrafish, for example, expresses the canonical $\operatorname{Tr} \beta 1$, homologous to the $\operatorname{TR} \beta 1$ of mammals and other vertebrates, and also an isoform with a 9 amino acid insert in the LBD that has been previously characterized and is able to bind T3 as well as the alternative TR ligand T2 (9). More surprisingly, we also detected a zebrafish isoform that has an up-stream putative alternative start site of transcription different from the other thrb isoforms that results in a Tr with an extended N-terminal fragment similar in length but not in sequence identity to that of the human $\operatorname{Tr} \beta 2$, which additionally contains the 9 amino acid insert plus 20 amino acids more located adjacently, probably generated by alternative splicing (Figure 2). This isoform, which we denominated $l$-trb+, is the largest thrb identified thus far in teleosts and not previously characterized in zebrafish. As in the case of thraa, the disruption of $l$-thrb + rendered larvae with deleterious effects upon symmetry and laterality that had not been previously described. In fact, the phenotype of both thraa and $l$-thrb + crispants was so strong that no detailed scrutiny was needed to identify affected larvae. Thus, one of the most interesting findings of the present study was the isoform-specific effects that thr exerted during development.

In concert with the phenotypic assessments, when gene expression was analyzed in crispant embryos, we identified early development genes, like sox10 and eve, that were specifically over-expressed in thraa and $l$-thrb + crispants, suggesting that for these genes, these specific thr isoforms function as transcription repressors. This would be in agreement with the prevailing concept that during early vertebrate development, TR act mainly as dominant negatives when unliganded, at least for TH positively regulated genes $(30,31)$. It is noteworthy that sox10 and eve are determinant for neural crest migration and tail extension $(41,42)$. Thus, precocious expression of sox10 and eve could explain, at least in part, the dramatic malformations observed in thraa and $l$-thrb + crispants. Interestingly, these two genes are regulated by THs in an opposite manner: T3 up-regulates sox10, while both, T2 and T3 down-regulate eve, showing the dynamic interplay between thr and ligands during early gastrulation. It would be very interesting for future experiments to isolate the different obtained alleles and look for differences or redundancy in its phenotype. Other genes that could be involved in these early developmental processes are zic and ets, which appear to be thraa- and l-thrb+-mediated, respectively. zic is involved in brain and somite development (43), while ets is a gene involved in vascularization (44). In contrast, with the exception of $m b p$, the expression of genes involved in the myelination process is not affected in the different crispants. The fact that $m b p$ is up-regulated in thrab crispants suggest a not myelinationrelated function of this gene in early development. As previously mentioned, these observations are in concert with the notion that myelination starts around $48 \mathrm{hpf}$ in zebrafish (27). The expression of other analyzed genes known to participate in zebrafish early development was not significantly modified. It is possible that these genes are not $\mathrm{TH}$ targets or that they act at different stages of development.

Overall, present results show that $\mathrm{TH}$ signaling participates in early zebrafish development. An interesting contribution of this study however is the identification of $\operatorname{Tr}$ isoformspecific mediated regulation of early gene expression. Thus, and although at this point, we cannot clearly decipher the respective contribution of each receptor isoform during early development, we did identify at least two genes whose regulation is specifically mediated by $\operatorname{Tr} \alpha$ and $\mathrm{L}-\operatorname{Tr} \beta+$, showing that the experimental strategies used in the present study will be useful to elucidate TR-specific functions.

\section{DATA AVAILABILITY}

All datasets generated for this study are included in the manuscript and/or the supplementary files.

\section{AUTHOR CONTRIBUTIONS}

IL and RR-O performed the CRISPR-Cas9 experiments. $\mathrm{PV}$ performed the qPCR experiments. $\mathrm{AO}$ and IL directly participated in the planning and execution of this study and drafted the manuscript. All authors provided critical comments to the manuscript and revised the text. All authors of this research paper have read and approved the final version submitted.

\section{FUNDING}

This study was supported by grant PAPIIT IN204517.

\section{ACKNOWLEDGMENTS}

We thank Jessica G. Norris for critically reviewing the manuscript. This work received technical support of Edith Espino Saldaña, Ramón Martínez Olvera, and Carlos Sair Flores Bautista.

\section{SUPPLEMENTARY MATERIAL}

The Supplementary Material for this article can be found online at: https://www.frontiersin.org/articles/10.3389/fendo. 2019.00156/full\#supplementary-material 


\section{REFERENCES}

1. Mansouri A, Chowdhury K, Gruss P. Follicular cells of the thyroid gland require Pax8 gene function. Nat Genet. (1998) 19:87-90. doi: $10.1038 / \mathrm{ng} 0598-87$

2. De Groef B, Grommen SVH, Darras VM. Hatching the cleidoic egg: the role of thyroid hormones. Front Endocrinol. (1992) 4:63.

3. Galton VA. The role of thyroid hormone in amphibian metamorphosis. Trends Endocrinol Metab. (1992) 3:96-100. doi: 10.1016/1043-2760(92)90020-2

4. Power DM, Llewellyn L, Faustino M, Nowell MA, Björnsson BT, Einarsdottir IE, et al. Thyroid hormones in growth and development of fish. Comp Biochem Physiol C Toxicol Pharmacol. (2001) 130:447-59. doi: 10.1016/S1532-0456(01)00271-X

5. Holzer G, Laudet V. Thyroid hormones and postembryonic development in amniotes. Curr Top Dev Biol. (2013) 103:397-425. doi: 10.1016/B978-0-12-385979-2.00014-9

6. Holzer G, Markov GV, Laudet V. Evolution of nuclear receptors and ligand signaling: toward a soft key-lock model? Curr Top Dev Biol. (2017) 125:1-38. doi: 10.1016/bs.ctdb.2017.02.003

7. Flamant F, Samarut J. Thyroid hormone receptors: lessons from knockout and knock-in mutant mice. Trends Endocrinol Metab. (2003) 14:85-90. doi: 10.1016/S1043-2760(02)00043-7

8. Lazcano I, Orozco A. Revisiting available knowledge on teleostean thyroid hormone receptors. Gen Comp Endocrinol. (2018) 265:128-32. doi: 10.1016/j.ygcen.2018.03.022

9. Mendoza A, Navarrete-Ramírez P, Hernández-Puga G, Villalobos P, Holzer G, Renaud JP, et al. 3,5-T2 is an alternative ligand for the thyroid hormone receptor $\beta 1$. Endocrinology. (2013) 154:2948-58. doi: 10.1210/en. 2013-1030

10. Kimmel CB, Ballard WW, Kimmel SR, Ullmann B, Schilling TF. Stages of embryonic development of the zebrafish. Dev Dyn. (1995) 203:253-310. doi: 10.1002/aja.1002030302

11. Stickney HL, Imai Y, Draper B, Moens C, Talbot WS. Zebrafish bmp4 functions during late gastrulation to specify ventroposterior cell fates. Dev Biol. (2007) 310:71-84. doi: 10.1016/j.ydbio.2007. 07.027

12. Blum M, Weber T, Beyer T, Vick P. Evolution of leftward flow. Semin Cell Dev Biol. (2009) 20:464-71. doi: 10.1016/j.semcdb.2008. 11.005

13. Takayama S, Hostick U, Haendel M, Eisen J, Darimont B. An Fdomain introduced by alternative splicing regulates activity of the zebrafish thyroid hormone receptor alpha. Gen Comp Endocrinol. (2008) 155:176-89. doi: 10.1016/j.ygcen.2007.04.012

14. Marelli F, Carra S, Agostini M, Cotelli F, Peeters R, Chatterjee K, et al. Patterns of thyroid hormone receptor expression in zebrafish and generation of a novel model of resistance to thyroid hormone action. Mol Cell Endocrinol. (2016) 424:102-17. doi: 10.1016/j.mce.2016.01.020

15. Chang J, Wang M, Gui W, Zhao Y, Yu L, Zhu G. Changes in thyroid hormone levels during zebrafish development. Zoolog Sci. (2012) 29:181-4. doi: $10.2108 / z s j .29 .181$

16. Essner JJ, Johnson RG, Hackett PB Jr. Overexpression of thyroid hormone receptor alpha 1 during zebrafish embryogenesis disrupts hindbrain patterning and implicates retinoic acid receptors in the control of hox gene expression. Differentiation. (1999) 65:1-11. doi: 10.1046/j.1432-0436.1999.6510001.x

17. Marelli F, Carra S, Rurale G, Cotelli F, Persani L. In vivo functional consequences of human THRA variants expressed in the zebrafish. Thyroid. (2017) 27:279-91. doi: 10.1089/thy.2016.0373

18. Vejnar CE, Moreno-Mateos MA, Cifuentes D, Bazzini AA, Giraldez AJ. Optimized CRISPR-Cas9 system for genome editing in zebrafish. Cold Spring Harb Protoc. (2016) 2016:prot086850. doi: 10.1101/pdb.prot 086850

19. Moreno-Mateos MA, Vejnar CE, Beaudoin JD, Fernandez JP, Mis EK, Khokha MK, et al. CRISPRscan: designing highly efficient sgRNAs for CRISPR-Cas9 targeting in vivo. Nat Methods. (2015) 12:982-8. doi: 10.1038/ nmeth.3543
20. Rosen JN, Sweeney MF, Mably JD. Microinjection of zebrafish embryos to analyze gene function. J Vis Exp. (2009) 25:1115. doi: 10.3791/1115

21. Meeker ND, Hutchinson SA, Ho L, Trede NS. Method for isolation of PCRready genomic DNA from zebrafish tissues. Biotechniques. (2007) 43:610-4. doi: $10.2144 / 000112619$

22. Hu Y, Xie S, Yao J. Identification of novel reference genes suitable for qRT-PCR normalization with respect to the zebrafish developmental stage. PLOS ONE. (2016) 11:e0149277. doi: 10.1371/journal.pone.0149277

23. Walpita CN, Van der Geyten S, Rurangwa E, Darras VM. The effect of 3,5,3'-triiodothyronine supplementation on zebrafish (Danio rerio) embryonic development and expression of iodothyronine deiodinases and thyroid hormone receptors. Gen Comp Endocrinol. (2007) J152:206-14. doi: 10.1016/j.ygcen.2007.02.020

24. Fraser TWK, Khezri A, Lewandowska-Sabat AM, Henry T, Ropstad E. Endocrine disruptors affect larval zebrafish behavior: testing potential mechanisms and comparisons of behavioral sensitivity to alternative biomarkers. Aquat Toxicol. (2017) 193:128-35. doi: 10.1016/j.aquatox.2017.10.002

25. Bertrand S, Thisse B, Tavares R, Sachs L, Chaumot A, Bardet PL, et al. Unexpected novel relational links uncovered by extensive developmental profiling of nuclear receptor expression. PLoS Genet. (2007) 3:e188. doi: 10.1371/journal.pgen.0030188

26. Rohde LA, Heisenberg CP. Zebrafish gastrulation: cell movements, signals, and mechanisms. Int Rev Cytol. (2007) 261:159-92. doi: 10.1016/S0074-7696(07)61004-3

27. Preston MA, Macklin WB. Zebrafish as a model to investigate CNS myelination. Glia. (2015) 63:177-93. doi: 10.1002/glia.22755

28. Orozco A, Navarrete-Ramírez P, Olvera A, García-G C. 3,5-Diiodothyronine (T2) is on a role. A new hormone in search of recognition. Gen Comp Endocrinol. (2014) 203:174-80. doi: 10.1016/j.ygcen.2014.02.014

29. Orozco A, Lazcano I, Hernández-Puga G, Olvera A. Non-mammalian models reveal the role of alternative ligands for thyroid hormone receptors. Mol Cell Endocrinol. (2017) 459:59-63. doi: 10.1016/j.mce.2017.03.003

30. Flamant F, Samarut J. Involvement of thyroid hormone and its alpha receptor in avian neurulation. Dev Biol. (1998) 197:1-11. doi: 10.1006/dbio. 1998.8872

31. Iskaros J, Pickard M, Evans I, Sinha A, Hardiman P, Ekins R. Thyroid hormone receptor gene expression in first trimester human fetal brain. J Clin Endocrinol Metab. (2000) 85:2620-3. doi: 10.1210/jcem.85.7.6766

32. Morvan-Dubois G, Fini JB, Demeneix BA. Is thyroid hormone signaling relevant for vertebrate embryogenesis? Curr Top Dev Biol. (2013) 103:365-96. doi: 10.1016/B978-0-12-385979-2.00013-7

33. Préau L, Fini JB, Morvan-Dubois G, Demeneix B. Thyroid hormone signaling during early neurogenesis and its significance as a vulnerable window for endocrine disruption. Biochim Biophys Acta. (2015) 1849:112-21. doi: 10.1016/j.bbagrm.2014.06.015

34. Bernal J. Thyroid hormone regulated genes in cerebral cortex development. J Endocrinol. (2017) 232:R83-97. doi: 10.1530/JOE-16-0424

35. Gothié JD, Demeneix B, Remaud S. Comparative approaches to understanding thyroid hormone regulation of neurogenesis. Mol Cell Endocrinol. (2017) 459:104-15. doi: 10.1016/j.mce.2017.05.020

36. Berbel P, Navarro D, Román GC. An evo-devo approach to thyroid hormones in cerebral and cerebellar cortical development: etiological implications for autism. Front Endocrinol. (2014) 5:146. doi: 10.3389/fendo.2014.00146

37. Glasauer SM, Neuhauss SC. Whole-genome duplication in teleost fishes and its evolutionary consequences. Mol Genet Genomics. (2014) 289:1045-60. doi: 10.1007/s00438-014-0889-2

38. Kawakami Y, Tanda M, Adachi S, Yamauchi K. cDNA cloning of thyroid hormone receptor betas from the conger eel, Conger myriaster. Gen. Compos. Endocrinol. (2003) 131:232-40. doi: 10.1016/S0016-6480(02)00638-X

39. Kawakami Y, Tanda M, Adachi S, Yamauchi K. Characterization of thyroid hormone receptor alpha and beta in the metamorphosing Japanese conger eel, Conger myriaster Gen. Comp. Endocrinol. (2003) 132:321-32. doi: 10.1016/S0016-6480(03)00087-X

40. Yaoita Y, Shi YB, Brown DD. Xenopus laevis alpha and beta thyroid hormone receptors. Proc Natl Acad Sci USA. (1990) 87:7090-4. doi: 10.1073/pnas.87.18.7090 
41. Joly JS, Joly C, Schulte-Merker S, Boulekbache H, Condamine H. The ventral and posterior expression of the zebrafish homeobox gene evel is perturbed in dorsalized and mutant embryos. Development. (1993) 119:1261-75.

42. Carney TJ, Dutton KA, Greenhill E, Delfino-Machín M, Dufourcq P, Blader P, et al. A direct role for Sox10 in specification of neural crestderived sensory neurons. Development. (2006) 133:4619-30. doi: 10.1242/ dev.02668

43. Rohr KB, Schulte-Merker S, Tautz D. Zebrafish zicl expression in brain and somites is affected by BMP and hedgehog signalling. Mech Dev. (1999) 85:147-59. doi: 10.1016/S0925-4773 (99)00044-1

44. Sumanas S, Lin S. Ets1-related protein is a key regulator of vasculogenesis in zebrafish. PLoS Biol. (2006) 4:e10. doi: 10.1371/journal.pbio. 0040010
Conflict of Interest Statement: The authors declare that the research was conducted in the absence of any commercial or financial relationships that could be construed as a potential conflict of interest.

The handling editor declared a past co-authorship with one of the authors AO.

Copyright (c) 2019 Lazcano, Rodríguez-Ortiz, Villalobos, Martínez-Torres, SolisSainz and Orozco. This is an open-access article distributed under the terms of the Creative Commons Attribution License (CC BY). The use, distribution or reproduction in other forums is permitted, provided the original author(s) and the copyright owner(s) are credited and that the original publication in this journal is cited, in accordance with accepted academic practice. No use, distribution or reproduction is permitted which does not comply with these terms. 